\title{
Pathophysiology of Spinal Cord Injuries and the Basis for Therapy
}

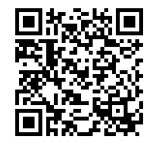

\author{
Jack Kushner* \\ George Washington University, USA
}

Submission: February 09, 2018; Published: February 16, 2018

*Corresponding author: Jack Kushner, George Washington University, Annapolis, Maryland 21409, USA, Email: jkaoportal@comcast.net

Abstract

This review of the pathophysiology of spinal cord injuries provides the backgound knowledge necessary for understanding the basis of the therapy for these patients.

Keywords: Spinal cord injuries; Pathophysiology spinal injuries

\section{Mini Review}

In order to understand just what happens when a patient experiences a spinal cord injury, one has to comprehend the injury pathophysiology. Then efforts can be made to interrupt the pathological processes in the neural, vascular and immune systems in order to stop the natural progression of events. If a patient has a transected or severely compressed and contused spinal cord secondary to an injury, the natural progression of events will lead to the loss of reflexes, the loss of sensation, and the paralysis of the affected limbs. So now we will proceed to discuss some of the cellular and molecular events after a spinal cord injury occurs, some of which are pathological and some are protective.

For the purposes of discussion, spinal cord injuries can be divided into three groups: (1) contusion, which is a bruising of the spinal cord, (2) laceration, which is a complete severing of the spinal cord, and (3) solid cord injury, which is an axon injury and a demyelination [1-5]. Immediately after a spinal cord injury in the acute phase, there is a systemic hypotension and a spinal shock along with hemorrhage. This leads to the death of the involved cells in the spinal cord from the injury and from ischemia or lack of blood. Swelling of the spinal cord then occurs along with major shifts in the body's electrolytes. Neurotransmitters also accumulate. The secondary phase is characterized by a continuation of the death of various cells, a continuation of the edema of the spinal cord, and a continuation of the shift of the electrolytes. Production of free-radicals follows. In addition, there is an occurrence of lipid peroxidation, a neutrophil and a lymphocyte invasion with a release of cytokines, an apoptosis which is a programmed cell death with an entry of calcium into the cells. The last phase or the chronic phase is marked by a continuation of the apoptosis, an alteration of the ion channels and receptors, a formation of fluid-filled cavities, scarring of the glial cells, demyelination, an attempt at regeneration of the neurons, and an alteration of the neurocircuits with a possible syringomyelia.

What is meant by spinal shock? In the acute phase after a severe spinal cord injury, the spinal cord cannot function properly due to a lack of oxygen and nutrients to the tissues. This lack of oxygen may be due to failure of the diaphragm to function if the injury is at C4 or due to an airway obstruction. This is all exacerbated by a prolonged hypotension due to acute blood loss. Another contributing factor might be the necrosis of the spinal cord neurons and the endothelial cells lining the spinal cord. Those neurons that do survive, respond with a barrage or a bombardment of electrical impulses known as action potentials. These, in turn, cause a major shift in the ions, which can cause other cellular membranes to rupture followed by a release of excessive amounts of neurotransmitters. Too many of the neurotransmitters release glutamate, which can be lethal to nearby neurons known as excitotoxicity. Glutamate also kills oligodendrocytes that surround and protect the axons. It is the death of the neurons that causes the losses of motor and sensory functions after the spinal cord injury (Faden AI, 1988). The spinal cord injury also causes damage to the small blood vessels of that cord which causes a disruption of the blood-spinal cord barrier, which normally protects the spinal cord. The blood-brain barrier controls the passage of cells and large molecules between the circulatory and the central nervous systems. It keeps immune system cells from entering the spinal cord. When the barrier is broken, immune system cells, mainly white blood cells, invade the spinal cord and causes an inflammatory response which produces an accumulation of fluid and allows more immune 
cells such as neutrophils, T-cells, macrophages, and monocytes to accumulate at the site of the injury. The immune system's response helps to fight off infection, but it sets off a release of cytokines, a group of molecules that are detrimental to the nerve cells. The microglia cells respond to the cytokines and beome macrophages and recruit additional microglia. The resting astrocytes express more cytokines and form scar tissue within the spinal cord. The swelling continues and the other surviving neurons are compressed and die. The remaining blood vessels narrow and some become thrombosed further aggravating the ischemia. This process causes the spinal cords gray matter to become necrotic more than the axons in the white matter of the cord NIH Publication No.03-160, pp.14-19).

Inflammation of the injured spinal cord also stimulates the production of free radicals. Normally these free radicals are a byproduct of normal cell metabolism. The spinal cord injury causes an overproduction of these free radicals, which attack and disable molecules crucial for cell function by modifying their structure. The free radicals cause a breakdown of the lipid metabolism within the cells, a process known as lipid peroxidation. They also change the behavior of other cells so that instead of protecting the injured patient, they turn into agents of destruction. Injury to the cells brings an influx of calcium into the cells, which promotes more free radical formation (See Spinal Cord Injury, $\mathrm{NIH)}$.

Some of the cells in the spinal cord die as a result of a programmed cell death called apoptosis or a cellular suicide. As a result, oligodendrocytes in the damaged areas of the spinal cord are killed which means that the cells forming the myelin for the axons are no longer available. Myelin is needed for the conduction of nerve impulses through the axons. This interferes with the communication of the body through the spinal cord to the brain. Thus the damaged spinal cord via the glial cells forms scar tissue, which makes it difficult if not impossible for the axons to transmit impulses. When the axons are injured and disconnected from their nerve cell bodies there follows a disintegration. This process is called Wallerian or orthograde degeneration. Nerve cell bodies with damaged axons and the axon segment that remains attached may die by retrograde degeneration. This degeneration begins at the site of the injury and progresses back toward the cell body.

When considering the various treatments and options for patients who have sustained a spinal cord injury, it must be remembered that surprisingly with incomplete lesions, there is a certain amount of spontaneous natural healing. Usually within 24 hours, various mechanisms aid the natural process of healing although this will by no means be complete. A few patients may even demonstrate a substantial degree of recovery [5-10]. It used to be thought that recovery would be limited and of short duration. Now the belief is that the healing process can continue for years. Thus treatments have been geared to this revised thinking.
The mature neurons that have been damaged will not recover. But because of the plasticity in the body and the spinal cord, surviving neurons can react and possibly adapt to change and compensate. It is this plasticity that allows the body to regenerate and regrow. It was assumed that neurons could only grow a few millimeters. However, Aguayo reported that central nervous system axons have the capacity to regrow long distances if they are not inhibited by molecules in their extracellular environment [8]. There are molecules in the extracellular environment that guide axon regrowth and if these molecules are lacking, then growth will be impeded. Axon growth can occur if the environment is supportive. In the peripheral nervous system, regrowth does occur because the Schwann cells are actively involved and support this activity.

Much of our knowledge about the natural healing in spinal cord injuries is derived from animal studies. For example, it is now known that remyelination by the Schwann cells and the oligodendrocytes occur and that there is a recovery of conduction in demyelinated axons. There is a strengthening of the existing synapses and that there is a sprouting of intact axons which form new circuits. Finally, growth factors are released and there is a shift of some function to alternate circuits [11-15]. This information has encouraged research in therapies that involve the transplantation of various cell types.

The fact that there is a limited regrowth and sprouting of axons to form new synapses are part of another mechanism of functional recovery [16]. Besides the growth factor molecules, there are other molecules that will guide the axons to their destination [17-18]. In order to plan for the treatment of spinal cord injuries, it is important to understand just how we move our extremities so that therapy can be directed toward restoring that lost function. To initiate and maintain movement in the extremities requires that a complex set of activities occur. There has to be the initial thought in the brain, which causes the brain's neurons to generate an action potential or lots of action potentials. The maintenance of these movements also requires neuron generating activity especially from different parts of the brain, such as the motor cortex, the basal ganglia, and the cerebellum into the brain stem. These potentials travel down the corticospinal tracts, down the final common pathway, to the peripheral nerves and muscles, which then contract. Some areas of the body require more cortical activity than do others: for example, a pianist who uses delicate finger movements requires more cortical input than does an uncomfortable sitting person who crosses his legs. These action potentials travel down the axons of the corticospinal tract to the ventral horn of the spinal cord. Signals must come from the motor cortex to modulate and regulate the actions of the muscles. These action potentials are also necessary to regulate the smooth muscles of many organs as well as the autonomic nervous system which regulate the blood pressure and body temperature.

Next it is important to understand that a feedback mechanism 
is regulated first from the sensory endings onto the posterior columns back to the cerebral cortex and the cerebellum. These actions occur mainly through the sensory roots and the posterior columns. Proprioception is moderated through this system and enables the patient to know at all times where his feet are, where his limbs might be, and how to adjust his movements to his environment. Other reflexes are handled through the reflex arc such as the patellar or ankle reflexes. This sensory information will bypass the posterior columns, will not go back to the brain, but will modulate through the reflex arc itself.

Why can some animals walk after a spinal cord injury when humans cannot? Why is it that spinal cord injury research on animals sometimes does not correlate with what we see with humans? Some experiments in the 1960s suggested that there is a central pattern generator (CPG), which is a complex circuit of neurons that regulate locomotion [19]. This generator is responsible for coordinated rhythmic muscle activity. The research was done on a cat when the brain stem was transected. No information could go from the spinal cord to the brain. After this transection, the cats could stand up on their own and walk. Similar results have been reported on transecting the spinal cords of rats and mice and all of these animals could still stand and walk. The conclusion was that the CPG in these animals was located in the spinal cord and not in the brain.

The question remains if the CPG is in the spinal cord and not in the brain, then why cannot humans stand on their own and walk after a spinal cord transection? The location of the CPG in these animals must be different than its location in humans. It may be that animals who walk on four legs have a different location for the CPG than humans who walk on two legs. Humans have more of a cortical dominance than the other animals studied [20]. Therefore, the results of research on animals is difficult if not impossible to translate into humans and primates. This is of concern when interpreting enthusiastic and hopeful research.

The majority of the spinal cord injured patients do experience spasticity, which is a state of increased muscular tone. This in turn can cause pain, flexion contractures, fractures, and even decubitus ulcers. Although the exact cause of this spasticity is not known, it is not for lack of theories. One theory is that it may be due to a greater excitability of the motor neurons and another states it may be due to injury induced sprouting of axons forming new synapses. Still another theory proposes that it may be due to a pathological alteration in the electrical properties of the motor neurons themselves.

Chronic pain after spinal cord injuries occurs in almost $80 \%$ of the patients. Several classifications have been attempted in an effort to better define the types of pain patients are experiencing. Sometimes the pain is such a problem that patients become depressed and thoughts of suicide are not uncommon in this group. The two largest categories of pain are nociceptive and neuropathic pain and there are sub classifications and subtypes of these. Nociceptive pain is that dull pain a patient experiences such as when a limb is fractured. Neuropathic pain is that pain which can be described as shooting, burning or electrical. Nociceptive pain can be treated with physical therapy, surgical therapy, and various pain medications. Neuropathic pain is more difficult to treat and may require surgical treatment such as neural decompression or fluid drainage.

In order to understand why patients with spinal cord injuries have problems urinating, we must review the anatomy of the urinary bladder and its relationship to the brain and the spinal cord. Two main muscles control bladder function: the detrusor muscle controls bladder contractions and the external sphincter muscles at the bladder base control bladder outflow. These muscles work in a reciprocal manner. When one contracts, the other relaxes and thus urine flows out of the bladder. These muscles are supplied by separate nerves and their coordination is done at the pons level in the brainstem. The pelvic nerve sends sensory messages back to the pons so that the person knows when the bladder is full and needs to be emptied. This is also called the voiding reflex. When a disruption of these spinal tracts above the sacral level occurs, this leads to a condition known as areflexia, when the detrusor muscle becomes flaccid. Thus the bladder does not empty and urine backs up to the kidneys. The bladder can become hyperreflexic and over reactive or the patient can have involuntary contractions of the sphincter muscles and cannot empty the bladder at all.

The central nervous system is essential to voluntary control of the gastrointestinal tract, particularly the elimination of feces. Problems in this area can lead to bowel obstruction and colorectal distention. Life- threatening hypertension can be triggered by bowel distension.

Many men and women who experience spinal cord injuries have reported that they have decreased libido or reduced sexual drive. Sexuality is partly controlled by various centers in the spinal cord. Also several brain regions such as the limbic center and the hypothalamic areas contribute to this as well [21]. The sexual response includes three separate functions: erection, ejaculation, and orgasm. Erection has two components controlled by distinct spinal cord reflexes. There is a psychogenic or mentally induced erection controlled by T11 to L2 segments and there is the reflexogenic erection controlled by the sacral cord. Ejaculation is controlled by that portion of the cord from T10-S4 and involves the sympathetic, parasympathetic and somatic nerves. Orgasm is the rhythmic pelvic floor contractions mediated by the sacral cord. Dysfuntions in either the brain, the gastro-intestinal or the urinary system can have an influence on the pleasurable aspects of the orgasm. For women, the spinal cord is also important since sexual response and arousal involve vaginal lubrication, swelling of the clitoris along with an increased heart and respiratory rate, and blood pressure. Psychogenic vaginal lubrication is controlled by T10-L2 and reflexive vaginal lubrication is controlled by S2S5. Only about $50 \%$ of women with a spinal cord injury are able 
to have an orgasm and researchers believe that the sacral reflex in necessary for orgasm to occur [22].

\section{References}

1. Bunge RP, Puckett WR, Becerra JL, Marcillo A, Quencer RM (1993) Observations on the pathology of human spinal cord injury. A review and classification of 22 new cases with details from a case of chronic cord compression with extensive focal demyelination. Advances in Neurology 59: 75-89.

2. Bunge RP, Puckett WR, Hiester EED (1997) Observations on the pathology of several types of human spinal cord injury, with emphasis on the astrocyte response to penetrating injuries. Advances in Neurology 72: 305-315

3. Faden AI, Simon RP (1988) A potential role for excitotoxins in the pathophysiology of spinal cord injury. Annals of Neurology 23 (6): 623-626.

4. Harper GP, Banyard PJ, Sharpe PC (1996) The International Spinal Research Trust's strategic approach to the development of treatments for the repair of spinal cord injury. Spinal Cord34(8): 449-459.

5. Hulsebosch CE (2002) Recent advances in pathophysiology and treatment of spinal cord injury. Advances in Physiology Education 26(1-4): 238-255.

6. Spinal Cord Injury prepared by Office of Scientific and Health Reports, National Institute of Neurological Disorders and Stroke, National Institutes of Health, Bethesda, Maryland, NIH Publication, pp.14-19

7. http://www.ninds.nih.gov/news_and_events/proceedings/sci_report. htm

8. Aguayo AJ, David S, Richardson P, Bray GM (1982) Axonal elongation in peripheral and central nervous system transplants. Advances in Cell Neurobiology 3: 215-234.

9. Alexander CJ, Sipski ML, Findley TW (1993) Sexual activities, desire, and satisfaction in males pre- and post-spinal cord injury. Archives of Sexual Behavior 22(3): 217-228.

10. Burchiel KJ, Hsu FP (2001) Pain and spasticity after spinal cord injury: Mechanisms and treatment. Spine 26(24 Suppl): S146-160.

11. Bunge MB, Wood PM (2004) Transplantation of Schwann cells and olfactory ensheathing cells to promote regeneration in the CNS. In Selzer ME, Clarke S, Cohen I.G. Dincan PW, Gage FH, eds. Textbook of Neural Repair and Rehabilitation. Cambridge, Cambridge University Press, United Kingdom, USA.

12. Fulton JA, Keller AD (1932) The Sign of Babinski: A Study of the Evolution of Cortical Dominance in Primates.

13. Hiersemenzel LP, Curt A, Dietz V (2000) From spinal shock to spasticity: Neuronal adaptations to a spinal cord injury. Neurology 54(8): 15741582.

14. Maynard FM, Karunas RS, Waring WP III (1990) Epidemiology of spasticity following traumatic spinal cord injury. Archives of Physical Medicine and Rehabilitation 71(8): 1999.

15. Rehabilitation pharmacotherapy: Preface. Physical Medicine \& Rehabilitation Clinics of North America 10(2): xv-xvi

16. Raineteau 0, Schwab ME (2001) Plasticity of motor systems after incomplete spinal cord injury. Nature Reviews Neuroscience 2(4): 263-273.

17. Shik ML, Severin FV, Orlovsky GN (1966) Control of walking and running by means of electric stimulation of the midbrain. Biofizika 11(4):659-666.

18. Shik ML, Severin FV, Orlovsky GN (1969) Control of walking and running by means of electric stimulation of the mesencephalon. Electroencephalography \& Clinical Neurophysiology 26(5): 549.

19. Sipski ML (2001) Sexual response in women with spinal cord injury: Neurologic pathways and recommendations for the use of electrical stimulation. Journal of Spinal Cord Medicine 24(3): 155-158.

20. Tator CH, MCCormick PC, Piepmeier JM, Benzel EC, Young W (1998) Biology of neurological recovery and functional restoration after spinal cord injury. Neurosurgery 42(4): 696-708.

21. Vilensky JA, O'Connor BL (1998) Stepping in nonhuman primates with a complete spinal cord transection: Old and new data, and implications for humans. Annals of the New York Academy of Sciences 860: 528530 .

22. Walsh FS, Doherty P (1997) Neural cell adhesion molecules of the immunoglobulin superfamily:Role in axon growth and guidance. Annual Review of Cell and Developmental Biology 13: 425-456.

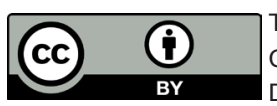

This work is licensed under Creative Commons Attribution 4.0 License DOI: 10.19080/CTBEB.2018.12.555835

Your next submission with Juniper Publishers
will reach you the below assets
- Quality Editorial service
- Swift Peer Review
- Reprints availability
- E-prints Service
- Manuscript Podcast for convenient understanding
- Global attainment for your research
- Manuscript accessibility in different formats
( Pdf, E-pub, Full Text, Audio)
- Unceasing customer service
Track the below URL for one-step submission
https://juniperpublishers.com/online-submission.php

